BULLETIN (New Series) OF THE

AMERICAN MATHEMATICAL SOCIETY

Volume 44, Number 4, October 2007, Pages 541-559

S 0273-0979(07)01178-0

Article electronically published on June 22, 2007

\title{
EULER AND ALGEBRAIC GEOMETRY
}

\author{
BURT TOTARO
}

Euler's work on elliptic integrals is a milestone in the history of algebraic geometry. The founders of calculus understood that some algebraic functions could be integrated using elementary functions (logarithms and inverse trigonometric functions). Euler realized that integrating other algebraic functions leads to genuinely different functions, elliptic integrals. These functions are not something ugly. As Abel discovered, their inverses are doubly periodic functions on the complex plane.

What we now call elliptic curves (algebraic curves of genus 1) take their name from elliptic integrals. Although these curves had been studied earlier, indeed in great depth by Fermat, it is Euler's analysis that clarifies the key points: elliptic curves are fundamentally different from rational curves, and not only in a negative way. They have a richer symmetry, the famous group structure possessed by an elliptic curve.

This paper considers two main themes in algebraic geometry descended from Euler's work: integrals of algebraic functions (in fancier terms, Hodge theory) and birational geometry. In section 1, we reach a major open problem of algebraic geometry: which representations of the fundamental group are summands of the cohomology of some family of algebraic varieties? Or, equivalently: which linear differential equations can be solved by integrals of algebraic functions? One might not expect any good answer to these questions, but in fact there are two promising approaches (the Simpson and Bombieri-Dwork conjectures).

Section 2 more elementary, gives an introduction to birational geometry. I hope to explain the significance of the problem of finite generation of the canonical ring, which has just been solved.

Thanks to Carlos Simpson for his comments on an earlier version.

\section{INTEGRALS OF ALGEBRAIC FUNCTIONS}

1.1. Elliptic integrals. Euler's main theorem on elliptic integrals, inspired by Fagnano's work on a special case, is the addition formula. Let $P(x)$ be a polynomial of degree 3 or 4 with distinct roots. An elliptic integral means the integral $\int_{a}^{b} F(x) d x / \sqrt{P(x)}$ for some rational function $F(x)$ [44, 22.7]. For simplicity, let us consider the elliptic integrals $\int_{a}^{b} d x / \sqrt{P(x)}$, which in modern language are the integrals of the differential form $\omega=d x / y$ along paths in the elliptic curve $y^{2}=P(x)$. Then Euler's addition formula says that

$$
\int_{0}^{p} \omega+\int_{0}^{q} \omega=\int_{0}^{p+q} \omega
$$

Received by the editors April 26, 2007.

2000 Mathematics Subject Classification. Primary 14C30; Secondary 14D05, 14E05. 
where $p+q$ refers to the group structure on the elliptic curve, which Euler wrote out as a rational function in the coordinates $(x, y)$. In retrospect, the addition formula follows from the invariance of the 1 -form $\omega$ with respect to the group structure of the elliptic curve. The general elliptic integrals $\int F(x) d x / \sqrt{P(x)}$ satisfy a similar addition formula modulo elementary functions.

When we work over the complex numbers, the integral $\int_{0}^{p} \omega$ depends on the choice of a homotopy class of paths from 0 to $p$ in the smooth compact elliptic curve $E(\mathbf{C}) \subset \mathbf{C P}^{2}$, and so it is really a function on the universal cover of $E(\mathbf{C})$. (The notation $X(k)$ means the set of solutions of a given set of algebraic equations over a field $k$. If the given polynomial $P(x)$ has real coefficients, then $E(\mathbf{R})$ is a real curve, whereas $E(\mathbf{C})$ is a complex curve and thus a real 2-manifold.) The addition formula says that the elliptic integral $\int_{0}^{p} \omega$ is a group homomorphism from the universal cover of $E(\mathbf{C})$ to the complex numbers. This homomorphism is in fact an isomorphism. Passing from the universal cover to the elliptic curve itself, we find that the group $E(\mathbf{C})$ is the quotient $\mathbf{C} /\left(\mathbf{Z} \omega_{1} \oplus \mathbf{Z} \omega_{2}\right)$, where $\omega_{1}$ and $\omega_{2}$ are the two periods of $\omega$, the integrals $\int_{\gamma_{1}} \omega$ and $\int_{\gamma_{2}} \omega$ along two closed loops $\gamma_{1}$ and $\gamma_{2}$ which form a basis for the homology group $H_{1}(E(\mathbf{C}), \mathbf{Z}) \cong H_{1}\left(\left(S^{1}\right)^{2}, \mathbf{Z}\right) \cong \mathbf{Z}^{2}$. By rescaling, this gives the description of a complex elliptic curve as $E(\mathbf{C})=$ $\mathbf{C} /(\mathbf{Z} \oplus \mathbf{Z} \tau)$ for some number $\tau$ in the upper half plane.

We can view Euler's theorem as a generalization of the properties of the logarithm function $\log p=\int_{1}^{p} d x / x$, which satisfies its own addition formula

$$
\log (p q)=\log p+\log q
$$

based on the group structure on another 1-dimensional algebraic group, the multiplicative group $\mathbf{C}^{*}$. As with elliptic functions, the logarithm is really defined on the universal cover of $\mathbf{C}^{*}$ and gives an isomorphism $\mathbf{C}^{*} \cong \mathbf{C} /(\mathbf{Z} \cdot 2 \pi i)$, where the number $2 \pi i$ enters as the integral of the 1 -form $d x / x$ over a closed loop around the origin in $\mathbf{C}^{*}$.

Elliptic integrals show the richness of integrals of algebraic functions. More general periods of integrals are central to Hodge theory, the main analytic technique for the study of algebraic varieties. The question of what sort of numbers arise as periods has also been a constant inspiration for transcendence theory [33, 41].

1.2. The Picard-Fuchs differential equation satisfied by a family of algebraic integrals. It is good to think about how the periods of an elliptic curve vary as a function of the coefficients. There are useful expositions by Carlson, MüllerStach, and Peters [8, 1.1] and Husemöller [26. Chapter 9]. Explicitly, consider the Legendre family of elliptic curves

$$
y^{2}=x(x-1)(x-\lambda)
$$

for $\lambda \in \mathbf{C}-\{0,1\}$. We can ask how the periods $\omega_{1}$ and $\omega_{2}$ of the 1 -form $\omega=d x / y$, with respect to some basis for $H_{1}\left(E_{\lambda}, \mathbf{Z}\right) \cong \mathbf{Z}^{2}$, vary as a function of $\lambda$. This makes sense because such a basis determines a basis for $H_{1}\left(E_{\mu}, \mathbf{Z}\right) \cong \mathbf{Z}^{2}$ for all nearby points $\mu \in \mathbf{C}-\{0,1\}$. Since $\omega=d x / \sqrt{x(x-1)(x-\lambda)}$, we have

$$
\omega^{\prime}:=\frac{\partial}{\partial \lambda} \omega=\frac{1}{2} \frac{d x}{\sqrt{x(x-1)(x-\lambda)^{3}}} .
$$


This is a rational 1-form on $E$ with a double pole at $p=(\lambda, 0)$, but its residue at $p$ vanishes, and so it represents an element of the de Rham cohomology $H_{D R}^{1}(E / \mathbf{C}) \cong$ $\mathbf{C}^{2}$.

Here $\omega$ and $\omega^{\prime}$ form a basis for $H_{D R}^{1}(E / \mathbf{C})$ for all $\lambda \neq 0,1$, and so the second derivative $\omega^{\prime \prime}$ must be a linear combination of $\omega$ and $\omega^{\prime}$ in $H_{D R}^{1}(E / \mathbf{C})$. Explicitly, we compute [8, 1.1.17] that

$$
\lambda(\lambda-1) \omega^{\prime \prime}+(2 \lambda-1) \omega^{\prime}+\frac{1}{4} \omega=0 .
$$

Equivalently, if we fix a basis $\gamma_{1}, \gamma_{2}$ for $H_{1}\left(E_{\lambda}, \mathbf{Z}\right)$, the periods $\omega_{1}=\int_{\gamma_{1}} \omega$ and $\omega_{2}=\int_{\gamma_{2}} \omega$ both satisfy the differential equation

$$
\lambda(\lambda-1) f^{\prime \prime}+(2 \lambda-1) f^{\prime}+\frac{1}{4} f=0,
$$

called the Picard-Fuchs equation of the Legendre family of elliptic curves.

This differential equation encodes many of the properties of the given family of elliptic curves. In a neighborhood of each point of $\mathbf{C}-\{0,1\}$, the solutions of the Picard-Fuchs equation form a two-dimensional vector space over the complex numbers. But following a local solution around a loop in $\mathbf{C}-\{0,1\}$ may transform it to a different solution. Thus the differential equation describes a flat bundle of rank 2 over $\mathbf{C}-\{0,1\}$, or equivalently a 2 -dimensional complex representation of the fundamental group $\pi_{1}(\mathbf{C}-\{0,1\})$, the free group on 2 generators. The representation is injective, with image conjugate to

$$
\Gamma:=\left\{\left(\begin{array}{ll}
a & b \\
c & d
\end{array}\right) \in S L(2, \mathbf{Z}): a \equiv c \equiv 1 \quad(\bmod 4), \quad b \equiv d \equiv 0 \quad(\bmod 2)\right\} .
$$

This is the monodromy group for the given family of elliptic curves, the image of $\pi_{1}(\mathbf{C}-\{0,1\})$ acting on $H_{1}\left(E_{\lambda}, \mathbf{Z}\right)$. Indeed, the Legendre family is the universal family of elliptic curves with the level structure described by $\Gamma$. From inspection of the differential equation, it would be hard to see that its monodromy group is a discrete subgroup of $G L(2, \mathbf{C})$, let alone a subgroup of $S L(2, \mathbf{Z})$. Figure 1 is Klein-Fricke's picture of a fundamental domain (the union of one white and one black triangle) for the action of $\Gamma$ on the unit disc $D$, yielding an isomorphism of $\mathbf{C}-\{0,1\}$ with the quotient $D / \Gamma$ [31, pp. 111-112].

The Picard-Fuchs equation for the Legendre family of elliptic curves is a special case of the Gauss hypergeometric differential equation:

$$
y^{\prime \prime}+\frac{(a+b-1) z-c}{z(z-1)} y^{\prime}+\frac{a b}{z(z-1)} y=0,
$$

with $a=b=1 / 2$ and $c=1$. Euler found the famous power-series solution on the unit disc around $z=0$ :

$$
F(a, b, c ; z)=1+\frac{a \cdot b}{1 \cdot c} z+\frac{a(a+1) \cdot b(b+1)}{1 \cdot 2 \cdot c(c+1)} z^{2}+\cdots .
$$

Euler also expressed the hypergeometric function as an integral [18, 27, Theorem 3.2.1]:

$$
F(a, b, c ; z)=\frac{\Gamma(c)}{\Gamma(a) \Gamma(c-a)} \int_{0}^{1} t^{a-1}(1-t)^{c-a-1}(1-t z)^{-b} d t .
$$

See Figure 2 for a picture of the monodromy of the hypergeometric differential equation in another nice case. 


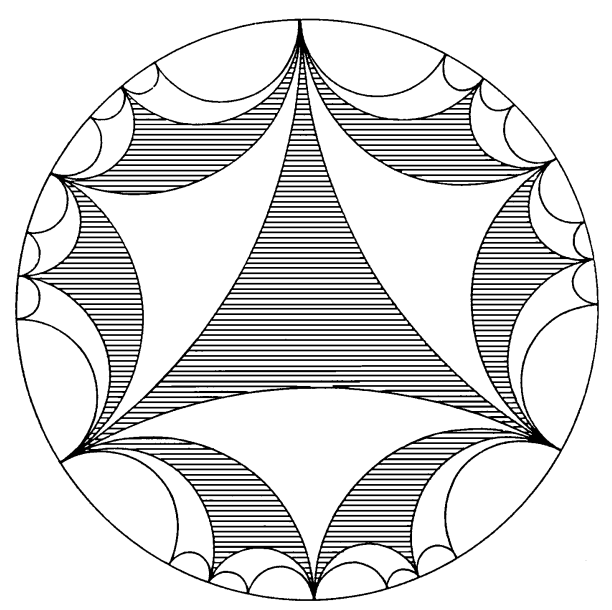

Figure 1. Monodromy group $\Gamma /\{ \pm 1\}$ in $P G L(2, \mathbf{C})$ for the hypergeometric differential equation with $(a, b, c)=(1 / 2,1 / 2,1)$.

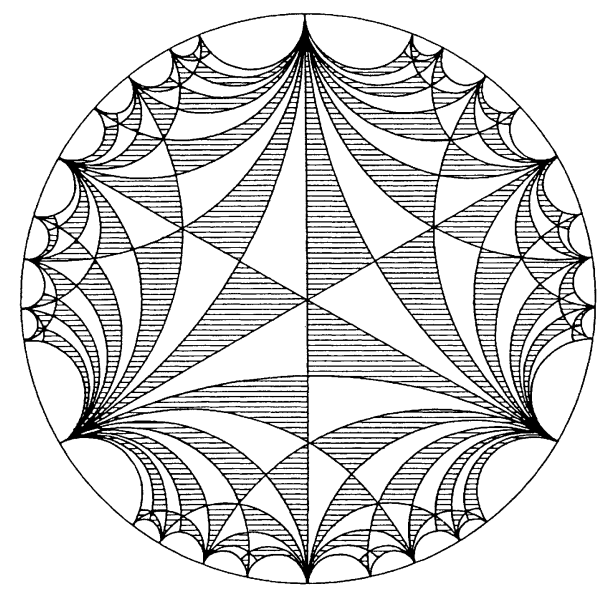

Figure 2. Monodromy group $S L(2, \mathbf{Z}) /\{ \pm 1\}$ in $P G L(2, \mathbf{C})$ for the hypergeometric differential equation with $(a, b, c)=$ $(1 / 12,1 / 12,2 / 3)$ [24, section 0.1].

The hypergeometric equation satisfied by the function $F(1 / 2,1 / 2,1 ; z)$, the Picard-Fuchs equation for the Legendre family of elliptic curves, is the simplest nontrivial example of a variation of Hodge structures [21, 43. We can first define the Hodge structure of a single elliptic curve $E$ over the complex numbers: it is the vector space $H_{D R}^{1}(E / \mathbf{C}) \cong \mathbf{C}^{2}$ together with the integral lattice $H^{1}(E, \mathbf{Z}) \cong \mathbf{Z}^{2} \subset$ $H_{D R}^{1}(E / \mathbf{C})$ and the linear subspace $H^{0}\left(E, \Omega^{1}\right) \cong \mathbf{C} \subset H_{D R}^{1}(E / \mathbf{C})$. For elliptic curves, as more generally for all smooth projective curves, the Hodge structure, essentially an object of linear algebra, determines the curve up to isomorphism (the Torelli theorem). 
A beautiful Picard-Fuchs equation is the one for the 1-parameter "Dwork family" of Calabi-Yau hypersurfaces $X_{\lambda}$ in $\mathbf{P}^{m-1}$ given by

$$
x_{1}^{m}+\cdots+x_{m}^{m}-m \lambda x_{1} \cdots x_{m}=0 .
$$

More precisely, we look at a summand of the cohomology, the invariants in the middle-dimensional cohomology $H^{m-2}\left(X_{\lambda}, \mathbf{Q}\right)$ under the obvious action of the group $(\mathbf{Z} / m)^{m-1}$. (Equivalently, we are looking at the cohomology of the quotient of $X_{\lambda}$ by this finite group.) This subspace of the cohomology has dimension $m-1$, and it has the unusually simple Hodge numbers $(1,1, \ldots, 1)$. (For $m=3, X_{\lambda}$ is an elliptic curve, and $H^{1}$ has Hodge numbers $(1,1)$.) We can define a nowherevanishing $(m-2)$-form on the $(m-2)$-dimensional variety $X_{\lambda}$ by

$$
\omega=\frac{d x_{1} \wedge \cdots \wedge d x_{m-2}}{x_{m-1}^{m-1}-\lambda x_{1} \ldots x_{m-2}}
$$

in coordinates with $x_{m}=1$. The Picard-Fuchs equation describing the periods of $\omega$ on the given summand of the cohomology of $X_{\lambda}$ is

$$
\left(\frac{d}{d \lambda}\right)^{m-1} y=\left(\lambda \frac{d}{d \lambda}\right)^{m-1}(\lambda y)
$$

29, Remark 2.3.8.5]. This is a generalized hypergeometric equation, with a power series solution around $\lambda=\infty$ given by

$$
G(\lambda)=\frac{1}{m \lambda} \sum_{a=0}^{\infty} \frac{(1 / m)_{a} \cdots((m-1) / m)_{a}}{a ! \cdots a !} \lambda^{-a m}
$$

29, Corollary 2.3.8.1], where $(a)_{n}$ means $a(a+1) \cdots(a+n-1)$.

The Dwork family of Calabi-Yau varieties (and specifically the summand of its cohomology we considered) has rich applications because it can be understood so explicitly. In the hands of Candelas, de la Ossa, Green and Parkes, this example inspired the whole phenomenon of mirror symmetry in string theory [7, 25]. The same family of hypersurfaces plays a starring role in number theory, as a generalization of elliptic curves. Using this family, Harris, Shepherd-Barron and Taylor were able to prove modularity for a broad class of Galois representations (not just of dimension 2), leading to the proof of the Sato-Tate conjecture for elliptic curves with nonintegral $j$-invariant [40, 22]. (The Sato-Tate conjecture, previously not known for any elliptic curve, is the following equidistribution statement. For an elliptic curve $E$ over $\mathbf{Q}$, Hasse showed that the number of $\mathbf{F}_{p}$-points of $E$ has the form $p+1-a_{p}$ where $\left|a_{p}\right| \leq 2 \sqrt{p}$. Write $a_{p}=2 \sqrt{p} \cos \theta_{p}$ where $\theta_{p}$ is between 0 and $\pi$. If $E$ does not have complex multiplication over the algebraic closure of $\mathbf{Q}$, then the Sato-Tate conjecture predicts that the angles $\theta_{p}$ are equidistributed with respect to the measure $(2 / \pi) \sin ^{2} \theta d \theta$ as $p$ varies.)

1.3. Rigid representations and Simpson's conjecture. It is a tantalizing problem to characterize the linear differential equations (or the representations of the fundamental group of a given base variety) that come from the cohomology of some family of algebraic varieties. This problem has the flavor of some other wildly difficult problems of mathematics, like the Fontaine-Mazur conjecture, which aims to characterize the representations of the Galois group $\operatorname{Gal}(\overline{\mathbf{Q}} / \mathbf{Q})$ that come from the cohomology of algebraic varieties over $\mathbf{Q}[19]$. To be precise, for an algebraic 
variety $U$, we try to characterize the complex representations of the fundamental group of $U$ that are summands of the cohomology with complex coefficients of some family of smooth projective varieties over $U$. Such representations are called motivic or of geometric origin.

It is equivalent to ask which linear differential equations with irreducible monodromy group have the property that their solutions are integrals of algebraic functions. (We allow definite integrals of an algebraic differential form over a domain in some $\mathbf{R}^{n}$ defined by algebraic equations and inequalities, where the function and the domain may depend algebraically on a point in the given variety. See Kontsevich-Zagier 33] for a related formulation.) For example, the Euler integral shows that the solutions of the hypergeometric equation can be written as integrals of the function $t^{a-1}(1-t)^{c-a-1}(1-t z)^{-b}$, which is algebraic if $a, b, c$ are all rational.

Technically, let us take a "differential equation" on a variety $U$ to mean an algebraic vector bundle with an algebraic flat connection over $U$. The monodromy of the solutions of a differential equation yields a representation of the fundamental group of $U$. The differential equations of geometric origin have regular singularities at infinity, which means that solutions have at most polynomial growth. Deligne's book on the topic is very readable [13, Theorem II.1.19, Theorem II.7.9]. Since the exponential function $e^{x}$ does not have polynomial growth at infinity, for example, the differential equation $y^{\prime}-y=0$ satisfied by $e^{x}$ has an irregular singularity at infinity. It follows that $e^{x}$ cannot be expressed as an integral of an algebraic function, in contrast to $\log x$.

The Riemann-Hilbert correspondence states that passing from differential equations with regular singularities over a smooth complex variety $U$ to complex representations of the fundamental group of $U$ is a one-to-one correspondence [13, Theorem 5.9]. As a result, we can casually identify the two objects.

The rationality of the coefficients $a, b, c$ in the hypergeometric equation generalizes to a property of all differential equations of geometric origin over a noncompact variety $U$ (in the hypergeometric case, $U$ is $\mathbf{C P}^{1}-\{0,1, \infty\}$ ). Think of $U$ as the complement of some codimension- 1 subvarieties $D_{1}, \ldots, D_{r}$ in a smooth projective variety. Then, for a differential equation of geometric origin (the de Rham cohomology of a family of varieties over $U$ with its flat connection or a summand of that), the eigenvalues of the monodromy on a small loop around each $D_{i}$ are roots of unity [13, Theorem III.2.3], 21], 28]. (The monodromy at infinity is said to be quasi-unipotent; "unipotent" would mean that all the eigenvalues were equal to 1.) For the hypergeometric differential equation, the eigenvalues of the monodromy are $\left(1, e^{2 \pi i(1-c)}\right)$ around $0,\left(1, e^{2 \pi i(c-a-b)}\right)$ around 1 , and $\left(e^{2 \pi i a}, e^{2 \pi i b}\right)$ around $\infty$. So these eigenvalues are roots of unity if and only if $a, b, c$ are rational. Thus quasiunipotence at infinity is enough to characterize which cases of the hypergeometric equation are of geometric origin.

A vast generalization of the hypergeometric differential equation is the class of rigid representations of the fundamental group of an algebraic variety. For a smooth variety $U$ over the complex numbers, possibly noncompact, we say that an irreducible representation $\rho: \pi_{1}(U) \rightarrow G L(n, \mathbf{C})$ is rigid if any nearby representation with the same eigenvalues on a small loop around each codimension-1 subvariety at infinity is conjugate to $\rho$. Riemann's study of the hypergeometric equation is based on its rigidity: the differential equation is determined by the conjugacy classes of 
the monodromy around 0,1 , and $\infty$, in the typical case where its monodromy is irreducible [27, Theorem 4.3.2].

Simpson conjectured:

Conjecture 1.1. Every rigid irreducible representation of the fundamental group of a smooth complex algebraic variety $U$, with quasi-unipotent monodromy at infinity in case $U$ is noncompact, is motivic.

The first result in this direction, still surprising, is Simpson's theorem that a rigid representation of the fundamental group of a smooth projective variety always comes from a complex variation of Hodge structures [38, Lemma 4.5]. Rigidity automatically implies that the representation maps into $G L(n, E)$ for some number field $E$. Conjecture 1.1 would imply that rigid representations actually map into $G L\left(n, O_{E}\right)$, where $O_{E}$ is a ring of integers, since for a family of smooth projective varieties, the fundamental group of the base acts on the integral cohomology of the fibers. In support of Conjecture 1.1, it is known that at primes where the representation is integral, it extends to a representation of the arithmetic fundamental group of some model of $U$ over a number field [38, Theorem 4].

The conjecture has been proved for $U$ of dimension 1 by Katz [30]. He gave an algorithm that produces all rigid representations of the fundamental group of a curve, the interesting ones being on $\mathbf{P}^{1}$ minus a finite set. If the monodromy at infinity (that is, around each point removed from $\mathbf{P}^{1}$ ) is quasi-unipotent, then Katz's construction shows that the representation is motivic, generalizing what happens for the hypergeometric equation on $\mathbf{P}^{1}-\{0,1, \infty\}$, essentially the only rank-2 example.

Recently Corlette and Simpson proved the main part of Conjecture 1.1 for 2dimensional representations with determinant 1 [1]. Namely, let $U$ be a smooth complex quasi-projective variety, and let $\rho: \pi_{1}(U) \rightarrow S L(2, \mathbf{C})$ be a representation with quasi-unipotent monodromy at infinity. Assume that $\rho$ is irreducible and remains so on all finite-index subgroups of $\pi_{1}(U)$. If $\rho$ is rigid, then it factors through a map $U \rightarrow Y$ with $Y$ a moduli space of abelian varieties with given endomorphisms. Here the Shimura varieties $Y$ that occur have as fundamental group (as an orbifold, in general) the unitary group of a hermitian form of dimension 2 over the ring of integers of some CM field. Such a group has obvious representations in $S L(2, \mathbf{C})$, yielding exactly the given representation of $\pi_{1}(U)$. This theorem is in fact more precise than Simpson's conjecture, where we expect certain representations of the fundamental group to come from some family of algebraic varieties, but we do not know what sort of varieties to expect. Here we get abelian varieties of a very precise kind.

1.4. The Bombieri-Dwork conjecture. There is another attempt to characterize the differential equations of geometric origin, the Bombieri-Dwork conjecture. Here there is no need to restrict to rigid representations of the fundamental group. The differential equation satisfied by the periods of an elliptic curve and the more general hypergeometric differential equation are model examples that helped to inspire the conjecture.

Consider a differential equation

$$
y^{(n)}+a_{n-1}(z) y^{(n-1)}+\cdots+a_{0}(z) y=0
$$


with $a_{j}(z)$ rational functions with coefficients in the field $\overline{\mathbf{Q}}$ of algebraic numbers. (More generally, the following definitions extend to differential equations on arbitrary smooth varieties over $\overline{\mathbf{Q}}$.) We say that the corresponding rank- $n$ vector bundle with flat connection over $\mathbf{P}^{1}$ minus a finite set is of geometric origin if there is an affine curve $C$ over $\overline{\mathbf{Q}}$ with a nonconstant map $C \rightarrow \mathbf{P}^{1}$ such that the bundle with connection pulled back to $C$ is a successive extension of summands of the de Rham cohomology of a family of smooth projective varieties over $C$ with its flat connection. On the other hand, we say that the flat connection is a $G$-connection if its solutions $\sum a_{n}\left(z-z_{0}\right)^{n}$ at some nonsingular point $z_{0} \in \overline{\mathbf{Q}}$, where we can assume $a_{n} \in \overline{\mathbf{Q}}$, are $G$-functions in the sense that the denominators of $a_{n}$ grow at most exponentially. By that we mean that there are positive integers $b_{n}$ such that $a_{i} b_{n}$ is an algebraic integer for all $i \leq n$ and $b_{n}$ is at most $C^{n}$ for some constant $C>0$. If this condition holds at one nonsingular point $z_{0} \in \overline{\mathbf{Q}}$, then it holds at all of them, by D. and G. Chudnovsky [16, section VIII.1]. There is a similar characterization of $G$-connections at a singular point, which is often easier to check in examples.

Every flat connection of geometric origin is a $G$-connection [2, Main Theorem]. For example, the equation $y^{\prime}-y=0$ is not a $G$-connection, because the denominators of its solution $e^{x}=\sum x^{n} / n$ ! grow too fast. This proves again that $e^{x}$ cannot be expressed as an integral of an algebraic function (over a domain depending algebraically on $x$ ).

Conjecture 1.2 (Bombieri-Dwork). Every G-connection on a smooth variety over $\overline{\mathbf{Q}}$ is of geometric origin [1, 2].

Among the evidence for Conjecture 1.2 is Katz's theorem that every $G$-connection has regular singularities at infinity and quasi-unipotent monodromy at infinity, both of which are well-known properties of the flat connection on the cohomology of a family of algebraic varieties [16, Theorem III.6.1]. (The theorem was initially stated under the slightly stronger assumption of "global nilpotence" [28, Theorem 13.0].) Conjecture 1.2 was proved for $G$-connections of rank 1 on any smooth variety by the Chudnovskys and André [10, [9, Théorème 3.5]. The rank-1 connections which are motivic are exactly those which are of finite order in the group of line bundles with connection, and so this theorem says that every rank-1 $G$-connection is of finite order.

The case of rank- 2 bundles over $\mathbf{P}^{1}$ minus 4 points is an intriguing test case for the Bombieri-Dwork conjecture. The only known cases seem to be where the monodromy group has an abelian subgroup of finite index, as happens for the "Lamé equation" with parameter $n$ a natural number [10, Theorem 7.2], 16. Example V.1.4].

It is tempting to bring in the analytic approach here. There is a hyperkahler moduli space of 2-dimensional representations of the fundamental group of $\mathbf{P}^{1}$ minus 4 points with given local monodromy and determinant 1 , which can be viewed as a complex affine cubic surface described by Fricke 20. What is the subspace consisting of complex variations of Hodge structure over $\mathbf{P}^{1}$ minus 4 points? Which of those are motivic? It may be useful to think about varying the 4 points while keeping the monodromy fixed: such variations are described by the famous Painlevé VI equation [27, a central object in the theory of integrable systems. Several of the known algebraic solutions of Painlevé VI are known to describe motivic representations of $\pi_{1}\left(\mathbf{P}^{1}-4\right.$ points) [23, 14, 15, 6, 4]. A case worth considering is where the eigenvalues of the monodromy at all four singular points are equal to 1 . 
Beauville's six families of semistable elliptic curves over $\mathbf{P}^{1}$ with 4 singular fibers give examples of motivic representations with local monodromy of that type [3].

\section{Birational GeOMetry}

2.1. The beginnings of birational geometry. The problem of birational classification of algebraic varieties is ancient: this is a fundamental problem we face in trying to understand algebraic equations. Indeed, users of algebraic geometry such as engineers often begin by hoping that every algebraic variety is rational, that is, that its points (outside some lower-dimensional subset) can be parametrized by affine space. Modern algebraic geometry begins with the realization that not every variety is rational: most simply, an elliptic curve is not rational, and this is reflected by the nontriviality of Euler's elliptic integrals. Let us consider how to prove that elliptic curves are not rational. The questions suggested by the proof lead naturally to minimal model theory, which has seen decisive advances in the past year.

We say that two algebraic varieties over a given field are birational if after removing a lower-dimensional subset from both varieties, they become isomorphic. The formulas defining a birational equivalence will be rational functions: the map may fail to be defined where these functions have poles. What makes it hard to determine whether two varieties are birational is that we have no idea how big the lower-dimensional subsets are that we might have to remove. Or, in algebraic terms, we do not know how high the degrees of the numerator and denominator of the rational functions might be. As a result, there is no known algorithm for determining whether two varieties are birational. (Is it possible to prove that there is no algorithm in full generality, say for varieties over the field $\overline{\mathbf{Q}}$ of algebraic numbers?)

In particular, we say that a variety is rational if it is birational to affine space (or equivalently to projective space) of some dimension. For example, every plane conic (a curve defined by an equation of degree 2, such as a circle, parabola, or hyperbola) is rational. (More precisely, a conic $C$ over a field $k$ is rational over $k$ if its set $C(k)$ of $k$-rational points is nonempty.) The proof by stereographic projection (attributed to Diophantus [42, I.X]) is simple. Choose a point $p$ on a conic $C$. Then consider the family of lines through $p$, which forms a projective line $\mathbf{P}^{1}$. Because $C$ has degree 2, a general line in this family will meet $C$ in 2 points, which must be $p$ and some other point. Conversely, a point on $C$ other than $p$ lies on a unique line through $p$, so we have constructed an isomorphism between $C$ minus a finite set and $\mathbf{P}^{1}$ minus a finite set. That is, $C$ is rational. (We need to remove finite sets if we start with a curve $C$ in the affine plane $A^{2}$; if we work in the projective plane $\mathbf{P}^{2}$, this map actually gives an isomorphism $C \cong \mathbf{P}^{1}$.)

For example, let us apply this proof to show that the circle $S^{1}=\left\{(x, y) \in A^{2}\right.$ : $\left.x^{2}+y^{2}=1\right\}$ is rational. The reader might like to compute the other point on the circle on the line through $p=(1,0)$ with slope $t$. We obtain a birational map $A^{1} \rightarrow S^{1}$ given by the formula

$$
t \mapsto\left(\frac{t^{2}-1}{t^{2}+1}, \frac{-2 t}{t^{2}+1}\right) .
$$

We see the appearance of rational functions. Notice that the proof works over any field, for example the rational numbers; thus this formula parametrizes all the pairs $(x, y)$ of rational numbers with $x^{2}+y^{2}=1$ (apart from $(1,0)$ ) by plugging in all 
the rational numbers $t$. This diophantine problem is not obvious until we see that the circle is a rational curve.

Euler used the rationality of conics $y^{2}=a x^{2}+b x+c$ to show that for every rational function $g(x, y)$, the integral $\int g\left(x, \sqrt{a x^{2}+b x+c}\right) d x$ can be expressed in terms of elementary functions. See Shafarevich's introduction to algebraic geometry [36. v. 1, p. 7; v. 2, Historical Sketch]. Euler felt the analogy between number theory and analysis, which we now call birational geometry, was a tantalizing possibility. In his words: "This shows how much more there is left to do in Diophantine Analysis, which beyond any doubt could contribute greatly to the whole of Analysis, finite and infinitesimal. In fact, one main tool of integral calculus consists in transforming irrational differential expressions into rational ones, and this comes directly from Diophantine Analysis, so that one has every right to expect further progress from that same study ..." [42, II.XVII], [17, p. 454].

In that paper, Euler proved the rationality of the cubic surface $x^{3}+y^{3}+z^{3}=w^{3}$ over the rational numbers by a "Diophantine method" extending the proof for conics. (The paper is called "A general solution of some Diophantine problems which seem to ordinary people to have only special solutions".) The key point is that over the quadratic field $\mathbf{Q}\left(\zeta_{3}\right)=\mathbf{Q}(\sqrt{-3})$, this surface contains two disjoint conjugate lines. A general line in $\mathbf{P}^{3}$ that meets each of those two lines will meet the cubic surface in exactly one other point, which gives the desired rational parametrization. See Kollár-Smith-Corti's introduction to birational geometry for details 32, Example 1.35].

The simplest examples of nonrational varieties are the smooth cubic curves, such as the Fermat cubic $x^{3}+y^{3}=z^{3}$ in $\mathbf{P}^{2}$; more generally, every smooth curve of degree at least 3 in $\mathbf{P}^{2}$ is not rational. Riemann's topological proof marks the beginning of modern algebraic geometry. A first step is to check that a birational map between smooth projective curves is in fact an isomorphism; all the hypotheses are needed here. So it suffices to show that a smooth curve of degree at least 3 in $\mathbf{P}^{2}$ is not isomorphic to $\mathbf{P}^{1}$. Riemann did this by considering these algebraic curves over the complex numbers as 2-dimensional real manifolds and computing that they are topologically different. Indeed, $\mathbf{C P}^{1}$ is homeomorphic to the 2 -sphere and hence has genus zero, whereas a smooth curve of degree $d$ has genus $(d-1)(d-2) / 2$, which is positive for $d$ at least 3 .

Unfortunately, this topological proof does not generalize well to higher dimensions. In complex dimension at least 2 , two smooth projective varieties which are birational need not be homeomorphic, as shown by the phenomenon of "blowing up", which changes the Betti numbers. The main topological invariant which is preserved by birational equivalences between smooth projective varieties is the fundamental group. There are many simply connected varieties, however, including all smooth complex hypersurfaces of dimension at least 2 in projective space. So the birational invariance of the fundamental group is of limited usefulness.

2.2. The canonical bundle. A better proof that smooth plane curves of degree at least 3 are not rational uses 1 -forms, as in the definition of elliptic integrals. For $n$-dimensional varieties, we use the line bundle of $n$-forms on $X$, called the canonical bundle. Let me try to say why the canonical bundle is so important in birational geometry. The fundamental point is that we can pull back differential forms. 
Let $f: X \rightarrow Y$ be a morphism of smooth algebraic varieties over a field $k$; it would be harmless to stick to the field of complex numbers. It is easy to define the derivative of a polynomial, and so we can talk about the derivative of $f$ : this is a linear map between the tangent spaces, $d f: T_{x} X \rightarrow T_{f(x)} Y$, for each point $x$ in $X$. This does not allow us to push forward vector fields, however; that is, there is no map " $f_{*}$ " from the global sections $H^{0}(X, T X)$ to $H^{0}(Y, T Y)$, as we see already in differential geometry. For example, if $f$ is not injective, the derivative of $f$ may well map a given vector field at different points in $X$ to different tangent vectors at the same point of $Y$.

But if we dualize, then we can pull back 1-forms, the sections of the dual to the tangent bundle, $\Omega_{X}^{1}=T^{*} X$. It is not so easy to say why this works, but it does: every morphism $f: X \rightarrow Y$ of smooth varieties determines a pullback map

$$
f^{*}: H^{0}\left(Y, \Omega_{Y}^{1}\right) \rightarrow H^{0}\left(X, \Omega_{X}^{1}\right),
$$

defined by the only formula that would make sense,

$$
f^{*}(\alpha)(v)=\alpha(d f(v))
$$

for any tangent vector $v$ at any point $x$ of $X$. More generally, as in differential geometry, we can pull back differential forms of any degree, defined as the exterior powers $\Omega_{X}^{i}=\Lambda^{i} \Omega^{1} X$. In particular, for $X$ smooth of dimension $n$, the line bundle $\Omega_{X}^{n}=\operatorname{det}\left(\Omega_{X}^{1}\right)$ is called the canonical bundle $K_{X}$ of $X$. At first sight, one might think that the dual line bundle, the anticanonical line bundle $-K_{X}=\operatorname{det}(T X)$, was more natural, but (as with the tangent bundle) we can neither pull back nor push forward sections of the anticanonical bundle in general. (Algebraic geometers commonly use additive notation for line bundles, so that $a K_{X}$, for any integer $a$, means the line bundle $K_{X}^{\otimes a}$.)

We can now prove the fundamental result linking the canonical bundle to birational geometry.

Theorem 2.1. A rational map between smooth projective varieties of the same dimension over a field, $f: X \rightarrow Y$, induces a pullback map on sections of the canonical bundle,

$$
f^{*}: H^{0}\left(Y, K_{Y}\right) \rightarrow H^{0}\left(X, K_{X}\right) .
$$

More generally, $f$ induces pullbacks

$$
f^{*}: H^{0}\left(Y, a K_{Y}\right) \rightarrow H^{0}\left(X, a K_{X}\right)
$$

for every integer $a \geq 0$.

For a birational map $f: X \rightarrow Y$, the pullback map $f^{*}: H^{0}\left(Y, a K_{Y}\right) \rightarrow$ $H^{0}\left(X, a K_{X}\right)$ is an isomorphism for every $a \geq 0$.

The restriction to $a \geq 0$ is unavoidable. For example, on the projective plane $\mathbf{P}^{2}$, the anticanonical bundle $-K_{X}$ is $3 H$ where $H$ is the hyperplane line bundle, and so $H^{0}\left(\mathbf{P}^{2},-K_{\mathbf{P}^{2}}\right)$ is the vector space of homogeneous cubic polynomials in 3 variables, which has dimension 10 . On the other hand, the space of sections of the anticanonical bundle on $\mathbf{P}^{1} \times \mathbf{P}^{1}$ is the tensor product of two copies of the vector space $H^{0}\left(\mathbf{P}^{1},-K_{\mathbf{P}^{1}}\right)=H^{0}\left(\mathbf{P}^{1}, 2 H\right)$ of dimension 3 , so it has dimension only 9 . Here $\mathbf{P}^{1} \times \mathbf{P}^{1}$ is birational to $\mathbf{P}^{2}$, since both contain the affine plane as an open subset. 
Proof. Let $f: X \rightarrow Y$ be a rational map between smooth projective varieties of the same dimension. By definition, this means that $f$ is a morphism from the complement of some lower-dimensional subset in $X$ to $Y$. The first step is to show that $f$ extends to the complement of some closed set of codimension at least 2 in $X$. Here is a naive method. We are assuming that $Y$ is projective, that is, a closed subvariety of $\mathbf{P}^{n}$ for some $n$. Therefore $f$ can be defined by $n+1$ rational functions on $X$,

$$
x \mapsto\left[f_{0}(x), \ldots, f_{n}(x)\right],
$$

not all zero. These functions are not uniquely determined; multiplying them all by the same rational function on $X$ (not identically zero) gives the same map to projective space.

It suffices to show that for every point $p$ in $X, f$ is defined outside a closed codimension-2 subset in a neighborhood of $p$. (We use the Zariski topology, which means that a closed subset is exactly the zero locus of some set of polynomial equations.) By multiplying $f_{0}, \ldots, f_{n}$ by a suitable rational function, we can assume that $f_{0}, \ldots, f_{n}$ are all regular functions (functions with no poles) in a neighborhood of $p$. Then our map is clearly defined outside the subset where $f_{0}=0, \ldots, f_{n}=0$. (At those points, the map is at first sight not defined, because $[0, \ldots, 0]$ is not a well-defined point in projective space.) We are done if the subset of $X$ defined by $f_{0}=0, \ldots, f_{n}=0$ has codimension at least 2 in a neighborhood of $p$. So suppose this closed set has codimension 1 near $p$. It then contains an irreducible subvariety $D$ of codimension 1 through $p$. The point is that because $X$ is smooth, a codimension- 1 subvariety is defined locally by just one equation, say $g=0$. Since $f_{0}, \ldots, f_{n}$ all vanish on the set where $g=0$, they must all be multiples of $g$ near $x$, so we can divide $f_{0}, \ldots, f_{n}$ by $g$ and still have regular functions that define our map $f$. By repeating the process if necessary, we can arrange that the common zero set of $f_{0}, \ldots, f_{n}$ has codimension at least 2 near $p$, as we want.

Thus, our rational map $f: X \rightarrow Y$ is defined by a morphism $f: U \rightarrow Y$ for some open subset $U$ of $X$ whose complement has codimension at least 2 . This gives us a pullback map

$$
f^{*}: H^{0}\left(Y, K_{Y}\right) \rightarrow H^{0}\left(U, K_{X}\right)
$$

and the same construction gives pullbacks

$$
f^{*}: H^{0}\left(Y, a K_{Y}\right) \rightarrow H^{0}\left(U, a K_{X}\right)
$$

for all $a \geq 0$. To finish the proof, it suffices to show that for any line bundle $L$ on $X$, every section of $L$ over $U$ extends (obviously uniquely) to a section of $L$ over $X$; that will give the desired pullback map

$$
f^{*}: H^{0}\left(Y, a K_{Y}\right) \rightarrow H^{0}\left(X, a K_{X}\right)
$$

for all $a \geq 0$.

It suffices to prove the statement about extending sections locally on $X$. That is, for each point $p$ in $X$ and any closed subset $S$ of codimension at least 2 in $X$, we have to show that a section of $L$ over a neighborhood of $p$ minus $S$ extends to the whole neighborhood of $p$. By definition of line bundles, we can choose a trivialization of $L$ on a neighborhood of $p$. So it suffices to show that a rational function $f / g$ on a neighborhood of $p$ which is regular outside a subset of codimension at least 2 is in fact regular. By the earlier argument, we can assume that $f$ and $g$ do not both vanish on any codimension-1 subvariety through $p$. Then our assumption gives that $g$ does not vanish on any codimension-1 subvariety through $p$, and hence that $g$ is 
nonzero at $p$, since the zero set of a regular function is a union of codimension-1 subvarieties. Thus $f / g$ is regular at $p$ as we want. (In complex analysis this is known as Hartogs's theorem.)

Finally, if we have a birational map $f: X \rightarrow Y$, then we have pullback maps associated to the rational maps $f$ and $f^{-1}$, and it is easy to deduce that the corresponding pullback maps on sections of $a K_{X}, a \geq 0$, are isomorphisms.

As we wanted, this implies that smooth plane curves of degree at least 3 are not rational. More generally, consider a hypersurface in projective space, meaning a closed subvariety of codimension 1 , or equivalently the subvariety defined by $f=0$ for some irreducible homogeneous polynomial $f$. The degree of $f$ is called the degree of the hypersurface. We have:

Corollary 2.2. Let $X$ be a smooth hypersurface of degree $d$ in $\mathbf{P}^{n+1}$ over a field. If $d \geq n+2$, then $X$ is not rational.

Proof. We begin by computing the canonical bundle of projective space $\mathbf{P}^{n}$. By definition, this is the line bundle of holomorphic $n$-forms, $K_{\mathbf{P}^{n}}=\Omega_{\mathbf{P}^{n}}^{n}$. On the open subset of points $\left[x_{0}, \ldots, x_{n}\right]$ in $\mathbf{P}^{n}$ with $x_{0} \neq 0$, we can scale to make $x_{0}=1$. In these coordinates, we can write down a rational section $\alpha$ of the canonical bundle by $\frac{d x_{1}}{x_{1}} \wedge \cdots \wedge \frac{d x_{n}}{x_{n}}$. A moment's calculation shows that this differential form looks the same, up to sign, in the other affine coordinate systems defined by setting $x_{i}=1$ for some $i$. Therefore, we read off that the $n$-form $\alpha$ has poles of order 1 at the $n+1$ hyperplanes $x_{0}=0, \ldots, x_{n}=0$ in $\mathbf{P}^{n}$, and it has no zeros. This means by the correspondence between divisors and line bundles that $K_{\mathbf{P}^{n}}=-(n+1) H$, where $H$ is the hyperplane line bundle. (A topologist would write instead that $c_{1}\left(T_{\mathbf{C P}^{n}}\right)=(n+1) c_{1}(H)$, where $c_{1}(H)$ is the generator of $H^{2}\left(\mathbf{C P}^{n}, \mathbf{Z}\right) \cong \mathbf{Z}$.)

For any smooth hypersurface $Y$ in a smooth variety $X$, we have an obvious exact sequence of vector bundles on $Y$,

$$
\left.0 \rightarrow T Y \rightarrow T X\right|_{Y} \rightarrow N_{Y / X} \rightarrow 0
$$

where the normal bundle $N_{Y / X}$ can be identified with the restriction of the line bundle $O(Y)$ to $Y$. (Here $O(Y)$ is the line bundle on $X$ whose first Chern class is represented by the codimension- 1 subvariety $Y$ of $X$.) Therefore, taking determinants of these vector bundles, we have an isomorphism of line bundles on $Y$ : $\left(-K_{X}\right)\left|Y \cong\left(-K_{Y}\right) \otimes O(Y)\right|_{Y}$, which is usually written in additive notation for line bundles as the adjunction formula:

$$
K_{Y}=\left.\left(K_{X}+Y\right)\right|_{Y}
$$

The adjunction formula lets us compute the canonical bundle of a hypersurface. In particular, for $X$ a hypersurface of degree $d$ in $\mathbf{P}^{n+1}$, we have

$$
\begin{aligned}
K_{X} & =\left.\left(K_{\mathbf{P}^{n+1}}+X\right)\right|_{X} \\
& =\left.(-(n+2) H+d H)\right|_{X} \\
& =\left.(d-n-2) H\right|_{X} .
\end{aligned}
$$

Therefore, if $X$ has degree $d \geq n+2, K_{X}$ is a nonnegative multiple of $H$, and so $H^{0}\left(X, K_{X}\right)$ is not zero. (In fact, it is exactly the vector space of homogeneous polynomials of degree $d-n-2$ in $n+2$ variables.) By contrast, $H^{0}\left(\mathbf{P}^{n}, a K_{\mathbf{P}^{n}}\right)=0$ for all $a>0$, because the canonical bundle of $\mathbf{P}^{n}$ is a negative multiple of the ample line bundle $H$. Thus $X$ is not birational to $\mathbf{P}^{n}$ for $d \geq n+2$. 
2.3. Varieties with positive, zero, or negative curvature. The proof of Corollary 2.2 suggests the central importance of the canonical bundle in algebraic geometry. In particular, the three extreme types of algebraic varieties are: Fano varieties, the varieties with ample anticanonical bundle; Calabi-Yau varieties, those with numerically trivial canonical bundle; and varieties with ample canonical bundle. (There is no standard name for the last class, but they are included in the larger class of varieties of general type.) For these three types of varieties $X$, the canonical line bundle is (respectively) of negative degree on all algebraic curves in $X$, zero on all curves, or positive on all curves. (Note that a complex manifold, in particular a complex curve, has a canonical orientation; that fact is needed for these signs to have an intrinsic meaning.) There are many varieties $X$ which are of mixed type in the sense that the canonical bundle $X$ is positive on some curves but not on others, but minimal model theory attempts to relate any variety to these three extreme types.

For example, the only Fano curve over the complex numbers is $\mathbf{P}^{1}$; the CalabiYau curves are the elliptic curves, and the curves with ample canonical bundle are the curves of genus at least 2. This division into three cases is famously related to curvature: the standard metric on $\mathbf{C P}^{1}=S^{2}$ has positive curvature, elliptic curves (being quotients $\mathbf{C} /\left(\mathbf{Z}^{2}\right)$ ) have flat metrics, and curves of genus at least 2 have metrics with negative curvature. These three classes generalize naturally to higher dimensions in terms of Ricci curvature. Namely, for complex manifolds with a Kähler metric (such as the restriction of the standard metric on $\mathbf{C P}{ }^{n}$ to any subvariety), the Ricci curvature can be identified with a real closed 2-form whose class in $H^{2}(X, \mathbf{R})$ is the first Chern class of the anticanonical bundle $-K_{X}$. And indeed Yau showed that, among all smooth complex projective varieties, the Fano varieties are exactly those admitting a Kähler metric with positive Ricci curvature, the Calabi-Yau varieties are those admitting a Ricci-flat Kähler metric, and the varieties with ample canonical bundle are those admitting a Kähler metric with negative Ricci curvature.

For real manifolds of dimension at least 3 , we cannot expect every manifold to have curvature with a fixed sign. In dimension 3, Perelman's geometrization theorem at least allows us to relate every 3-manifold to those with curvature of a fixed sign, and it makes clear that "most" 3-manifolds are hyperbolic. There is no hope of generalizing these ideas to real manifolds of higher dimensions. Rather amazingly, in algebraic geometry these ideas do generalize to all dimensions, according to the minimal model conjecture. That is, we can relate all varieties to the three extreme types: Fano varieties, Calabi-Yau varieties, and varieties with ample canonical bundle. Enough of the conjecture is now known to make the story convincing.

A weak analogue of this trichotomy which works for arbitrary varieties is given by the Kodaira dimension. The Kodaira dimension of a smooth projective variety $X$ is the most basic birational invariant, measuring the growth of the vector spaces $H^{0}\left(X, a K_{X}\right)$ of "pluricanonical forms" as a goes to infinity. We say that $X$ has Kodaira dimension $-\infty$ if the spaces $H^{0}\left(X, a K_{X}\right)$ are 0 for all $a>0$, as happens in the case of projective space. Otherwise, the Kodaira dimension of $X$ is the unique integer $m$ such that the dimension of $H^{0}\left(X, a K_{X}\right)$ grows like a constant times $a^{m}$ for all multiples $a$ of some fixed positive integer. The Kodaira dimension divides all varieties of dimension $n$ into $n+1$ classes, those of Kodaira dimension $-\infty, 0,1, \ldots$, 
or $n$. A variety $X$ is defined to be of general type if its Kodaira dimension is equal to its dimension, the maximum possible value. Informally, most varieties are of general type, as we saw in the case of smooth hypersurfaces in projective space: those of degree greater than $n+2$ in $\mathbf{P}^{n+1}$ are of general type and in fact have the stronger property that the canonical bundle is ample.

There is a definitive result on the birational classification of varieties with ample canonical bundle, that is, for the varieties with negative curvature:

Theorem 2.3. If two smooth projective varieties with ample canonical bundle over a field $k$ are birational, then they are isomorphic.

Proof. For any smooth projective variety $X$ over a field $k$, we define the canonical ring of $X$ to be the direct sum

$$
\oplus_{a \geq 0} H^{0}\left(X, a K_{X}\right)
$$

which is a graded algebra over $k$. More generally, for any line bundle $L$, let us write $R(X, L)$ for the ring $\oplus_{a \geq 0} H^{0}(X, a L)$. For any rational map $f: X \rightarrow Y$ between varieties of the same dimension, the pullback map

$$
f^{*}: R\left(Y, K_{Y}\right) \rightarrow R\left(X, K_{X}\right)
$$

constructed in Theorem 2.1 is a ring homomorphism, as is immediate from the definition. Therefore, if two smooth projective varieties are birational, then their canonical rings are isomorphic.

Next, we note that for any ample line bundle $L$ on a projective variety $X$, we can reconstruct the variety $X$ from the graded ring $R(X, L)$, the standard notation being that $X=\operatorname{Proj} R(X, L)$. We recall briefly what the projective variety $\operatorname{Proj} R$ means, for any finitely generated commutative graded algebra $R$ over a field $k$. First find a positive integer $a$ such that the vector space $R_{a}$ in degree $a$ generates the ring $R$ in all degrees a multiple of $a$. Then Proj $R$ will be a subvariety of the projective space associated to the vector space $R_{a}$ of generators. We then find the relations that define the ring $R$ (in degrees a multiple of $a$ ); these give the equations that define the projective variety Proj $R$.

Thus, if $X$ and $Y$ are birational varieties with ample canonical bundle, then we have

$$
X \cong \operatorname{Proj} R\left(X, K_{X}\right) \cong \operatorname{Proj} R\left(Y, K_{Y}\right) \cong Y,
$$

using the birational invariance of the canonical ring.

The corresponding statement is far from true for varieties with ample anticanonical bundle (Fano varieties). For example, $\mathbf{P}^{2}$ and $\mathbf{P}^{2}$ blown up at a point both have ample anticanonical bundle, and they are birational, but they are not isomorphic. Again, the canonical bundle behaves better than the anticanonical bundle.

2.4. The canonical model. When you blow up a point $p$ on a smooth variety $X$ of dimension $n$, you obtain a new smooth variety $Y$ with a proper birational morphism $f: Y \rightarrow X$, in which the point $p$ is replaced by the projective space $\mathbf{P}^{n-1}$ of lines in the tangent space to $X$ at $p$. If $X$ has dimension $n$ at least 2 (otherwise blowing up does nothing), then the canonical bundle $K_{Y}$ has negative degree (namely $-(n-1)$ ) on a line $\mathbf{P}^{1} \subset E \cong \mathbf{P}^{n-1}$. In short, every blown-up variety contains a $K$-negative curve. So if you blow up a point on a variety with ample canonical bundle, you still get a variety of general type because that property is birationally invariant, 
but you lose the property that the canonical bundle is ample. (This is inevitable in view of Theorem 2.3.)

This leads to the question: could it be that every variety $X$ of general type is birational to a variety with ample canonical bundle, called the canonical model of $X$ ? The canonical model is unique if it exists by Theorem 2.3. If that were true, it would reduce the problem of birational classification for varieties of general type to problems of classifying varieties with ample canonical bundle up to isomorphism, which is elementary by comparison.

It follows from Theorem 2.3 that every variety $X$ of general type is birational to at most one variety $Y$ with ample canonical bundle. In fact, the proof makes clear what $Y$ must be: it must be Proj of the canonical ring of $X$. But there are two obstacles here: (1) If the canonical ring of $X$ is not finitely generated as a $k$-algebra, then Proj does not make sense, and $X$ has no canonical model. (2) Even if the canonical ring of $X$ is finitely generated, the corresponding projective variety may be singular, as we see in examples. In that case, $X$ has no smooth canonical model.

Issue (2) was understood by 1980 . We simply have to accept canonical models with singularities. Moreover, the singularities that occur on canonical models of varieties of general type are not bad: they are exactly the canonical singularities defined by Reid. His recent memoir is recommended as a survey [35.

For example, the canonical singularities of surfaces are the famous ADE singularities, the quotient singularities $\mathbf{C}^{2} / G$ for finite subgroups $G$ of $S L(2, \mathbf{C})$. In particular, the simplest canonical singularity is $\mathbf{C}^{2} /\{ \pm 1\} \cong\left\{(x, y, z) \in \mathbf{C}^{3}: x y=z^{2}\right\}$, the surface node.

We can pull back sections of the canonical bundle (and its positive powers) for varieties with canonical singularities. As a result, the proof that the canonical ring is a birational invariant generalizes from smooth projective varieties to projective varieties with canonical singularities. This justifies the definition: a canonical model of a variety $X$ of general type is a projective variety $Y$ birational to $X$ which has canonical singularities and ample canonical bundle. A canonical model is unique if it exists, since the proof of Theorem 2.3 works for varieties with canonical singularities. Indeed, Reid proved:

Theorem 2.4. Let $X$ be a smooth projective variety of general type. The following are equivalent:

(1) $X$ has a canonical model, which is unique if it exists.

(2) The canonical ring of $X$ is finitely generated.

This shows the fundamental importance of determining whether the canonical ring is finitely generated. This was proved by Mumford 45] for surfaces in any characteristic, by Mori [34] for 3-folds, and by Shokurov for 4-folds (see 37] and the new book on flips for 3-folds and 4-folds [12]). It is now known in all dimensions. Many of the ideas were introduced by Shokurov, who has been pushing the minimal model program forward for many years by finding inductive approaches to the problem.

Theorem 2.5 (Birkar-Cascini-Hacon-McKernan [5, 2006; Siu [39], 2006). Let X be a smooth projective variety over a field of characteristic zero. Then the canonical ring of $X$ is finitely generated. 
As a result, every variety of general type over a field of characteristic zero has a unique canonical model.

Thus the problem of birational classification for varieties of general type (the biggest class of algebraic varieties) reduces to the problem of classifying varieties with ample canonical bundle (and canonical singularities) up to isomorphism. This is a much easier problem; for example, it is a finite problem to check whether two varieties with ample canonical bundle over an algebraically closed field are isomorphic. So we now have an algorithm to determine whether two varieties of general type are birational.

The problem of birational classification is harder for varieties of Kodaira dimension less than the dimension and much harder for varieties of Kodaira dimension $-\infty$ such as Fano varieties. Determining exactly which Fano varieties (for example, which hypersurfaces of low degree) are rational looks like the hardest problem in birational geometry in dimensions at least 3 despite many recent advances.

\section{ABOUt THE AUTHOR}

Burt Totaro is Lowndean Professor of Astronomy and Geometry at the University of Cambridge. He is a winner of the Prix Franco-Britannique and was an invited speaker at the 2002 ICM in Beijing.

\section{REFERENCES}

[1] Y. André. G-functions and geometry. Vieweg (1989). MR990016 (90k:11087)

[2] Y. André and F. Baldassarri. Geometric theory of $G$-functions. Arithmetic geometry (Cortona, 1994), 1-22. Cambridge (1997). MR1472489 (99c:12011)

[3] A. Beauville. Les familles stables de courbes elliptiques sur $\mathbf{P}^{1}$ admettant quatre fibres singulières. C. R. Acad. Sci. Paris 294 (1982), 657-660. MR664643 (83h:14008)

[4] B. Ben Hamed and L. Gavrilov. Families of Painlevé VI equations having a common solution. Int. Math. Res. Not. 2005, no. 60, 3727-3752. MR2205113

[5] C. Birkar, P. Cascini, C. Hacon, and J. McKernan. Existence of minimal models for varieties of log general type. arXiv:math.AG/0610203

[6] P. Boalch. From Klein to Painlevé via Fourier, Laplace and Jimbo. Proc. London Math. Soc. 90 (2005), 167-208. MR2107041 (2005h:34233)

[7] P. Candelas, X. de la Ossa, P. Green, and L. Parkes. A pair of Calabi-Yau manifolds as an exactly soluble superconformal theory. Nuclear Phys. B. 359 (1991), 21-74. MR 1115626 (93b:32029)

[8] J. Carlson, S. Müller-Stach, and C. Peters. Period mappings and period domains. Cambridge (2003). MR2012297 (2005a:32014)

[9] A. Chambert-Loir. Théorèmes d'algébricité en géométrie diophantienne (d'après J.-B. Bost, Y. André, D. \& G. Chudnovsky). Séminaire Bourbaki 2000/2001, Astérisque 282 (2002), 175-209. MR 1975179 (2004f:11062)

[10] D. Chudnovsky and G. Chudnovsky. Applications of Padé approximations to the Grothendieck conjecture on linear differential equations. Number theory (New York, 1983-84), 52-100. LNM 1135, Springer (1985). MR803350 (87d:11053)

[11] K. Corlette and C. Simpson. On the classification of rank two representations of quasiprojective fundamental groups. arXiv:math.AG/0702287

[12] A. Corti, ed. Flips for 3-folds and 4-folds. Oxford (2007).

[13] P. Deligne. Equations différentielles à points singuliers régulières. LNM 163, Springer (1970). MR0417174 (54:5232)

[14] B. Dubrovin. Painlevé transcendents in two-dimensional topological field theory. The Painlevé property, 287-412. Springer (1999). MR1713580(2001h:53131) 
[15] B. Dubrovin and M. Mazzocco. Monodromy of certain Painlevé-VI transcendents and reflection groups. Invent. Math. 141 (2000), 55-147. MR1767271 (2001j:34114)

[16] B. Dwork, G. Gerotto and F. Sullivan. An introduction to G-functions. Princeton (1994). MR.1274045 (96c:12009)

[17] L. Euler. Solutio generalis quorundam problematum Diophanteorum quae vulgo nonnisi solutiones speciales admittere videntur. Leonhardi Euleri opera omnia, v. 1, pt. 2, 428-458. Teubner (1915).

[18] L. Euler. Introduction to analysis of the infinite, 2 vols. Springer (1988, 1990). MR715928 (85d:01030)

[19] J.-M. Fontaine and B. Mazur. Geometric Galois representations. Elliptic curves, modular forms, \& Fermat's last theorem (Hong Kong, 1993), 41-78. International Press (1995). MR:1363495 (96h:11049)

[20] W. Goldman and W. Neumann. Homological action of the modular group on some cubic moduli spaces. Math. Res. Lett. 12 (2005), 575-591. MR2155233(2006h:57015)

[21] P. Griffiths. Periods of integrals on algebraic manifolds: summary of main results and discussion of open problems. Bull. AMS 75 (1970), 228-290. MR0258824 (41:3470)

[22] M. Harris, N. Shepherd-Barron, and R. Taylor. A family of Calabi-Yau varieties and potential automorphy. Preprint (2006).

[23] N. Hitchin. Poncelet polygons and the Painlevé equations. Geometry and analysis (Bombay, 1992), 151-185. Tata Inst. Fund. Res. (1995). MR1351506 (97d:32042)

[24] R.-P. Holzapfel. Geometry and arithmetic around Euler partial differential equations. D. Reidel (1986). MR0867406 (88b:32075)

[25] K. Hori, S. Katz, A. Klemm, R. Pandharipande, R. Thomas, C. Vafa, R. Vakil, and E. Zaslow. Mirror symmetry. AMS (2003). MR2003030 (2004g:14042)

[26] D. Husemöller. Elliptic curves. Springer (2004). MR2024529(2005a:11078)

[27] K. Iwasaki, H. Kimura, S. Shimomura, and M. Yoshida. From Gauss to Painlevé: a modern theory of special functions. Vieweg (1991). MR.1118604(92j:33001)

[28] N. Katz. Nilpotent connections and the monodromy theorem: applications of a result of Turrittin. Publ. Math. IHES 39 (1970), 175-232. MR0291177 (45:271)

[29] N. Katz. Algebraic solutions of differential equations ( $p$-curvature and the Hodge filtration). Invent. Math. 18 (1972), 1-118. MR0337959 (49:2728)

[30] N. Katz. Rigid local systems. Princeton (1996). MR.1366651 (97e:14027)

[31] F. Klein and R. Fricke. Vorlesungen über die Theorie der elliptischen Modulfunktionen, v. 1. Teubner (1890).

[32] J. Kollár, K. Smith, and A. Corti. Rational and nearly rational varieties. Cambridge (2004). MR2062787(2005i:14063)

[33] M. Kontsevich and D. Zagier. Periods. Mathematics unlimited, 771-808. Springer (2001). MR1852188 (2002i:11002)

[34] S. Mori. Flip theorem and the existence of minimal models for 3-folds. J. Amer. Math. Soc. 1 (1988), 117-253. MR924704 (89a:14048)

[35] M. Reid. Twenty-five years of 3-folds - an old person's view. Explicit birational geometry of 3-folds, 313-343. Cambridge (2000). MR1798985 (2002b:14001)

[36] I. Shafarevich. Basic algebraic geometry, 2 vols. Springer (1994). MR.1328834 (95m:14002)

[37] V. Shokurov. Prelimiting flips. Tr. Mat. Inst. Steklova 240 (2003), 82-219. MR.1993750 (2004k:14024)

[38] C. Simpson. Higgs bundles and local systems. Publ. Math. IHES 75 (1992), 5-95. MR.1179076 (94d:32027)

[39] Y.-T. Siu. A general non-vanishing theorem and an analytic proof of the finite generation of the canonical ring. arXiv:math.AG/0610740

[40] R. Taylor. Automorphy for some $l$-adic lifts of automorphic mod $l$ Galois representations. II. Preprint (2006).

[41] M. Waldschmidt. Transcendence of periods: the state of the art. Pure Appl. Math. Q. 2 (2006), 435-463. MR2251476 (2007d:11083)

[42] A. Weil. Number theory: an approach through history. Birkhäuser (1984). MR734177 (85c:01004)

[43] C. Voisin. Hodge theory and complex algebraic geometry, 2 vols. Cambridge $(2002,2003)$. MR:1988456(2005c:32024a) 
[44] E. Whittaker and G. Watson. A course of modern analysis. Cambridge (1962). MR0178117 $(31: 2375)$

[45] O. Zariski. The theorem of Riemann-Roch for high multiples of an effective divisor on an algebraic surface. Appendix by D. Mumford. Ann. Math. 76 (1962), 560-615. MR0141668 $(25: 5065)$

Department of Pure Mathematics and Mathematical Statistics, Wilberforce Road, Cambridge CB3 0WB, England

E-mail address: b.totaro@dpmms.cam.ac.uk 